\title{
Polynomial System Satisfying a Special Functional Equation
}

\author{
By A. M. Chak
}

A system of polynomials $\left\{P_{n}(x)\right\}$ is called an Appell set, if

$$
P_{n}^{\prime}(x)=P_{n-1}(x) \quad(n=0,1,2, \cdots) .
$$

Nielsen [2] considered a remarkable subset of the Appell set by considering a set of polynomials which satisfy the two functional equations,

$$
P_{n}^{\prime}(x)=P_{n-1}(x) \text { and } P_{n}(-x-1)=(-1)^{n} P_{n}(x)
$$

for $n=0,1,2, \cdots$; he has shown their importance in the theory of Bernoulli and Euler numbers. Later Ward [4] generalized the set by considering a set of polynomials $\left\{Y_{n}(x)\right\}$ for which

$$
Y_{n}^{\prime}(x)=Y_{n-1}(x) \quad \text { and } \quad Y_{n}(a x+b)=\tau_{n} Y_{n}(x)
$$

for $n=0,1,2, \cdots$ and $a$ and $b$ are any complex numbers. More recently Sharma and Chak* [3] studied a class of polynomials $\left\{H_{n}(x)\right\}$ in $x$, such that for $n=$ $0,1,2, \cdots$

$$
D_{q}\left\{H_{n}(x)\right\}=H_{n-1}(x) \text { where } \quad D_{q} f(x)=\frac{f(q x)-f(x)}{(q-1) x} .
$$

Immediately after, a paper by Carlitz [1] appeared studying various polynomials related to Theta functions. It was this paper of Carlitz which suggested, as a natural study, the systems of polynomials which satisfy a functional equation of the form

$$
D_{q}\left\{H_{n}(x)\right\}=H_{n-1}\left(q^{k} x\right) \quad \text { where } k \text { is a real number, }
$$

and $n=0,1,2, \cdots ;$ and also to examine some subsets of this class of polynomials which have properties analogous to regular and cyclic sets of Nielsen [2] and of Ward [4].

1. $(q, k)$-Harmonic Sequences. If $H_{n}(x)=\sum_{i=0}^{n} a_{i} x^{n-i}$, we put

$$
\begin{aligned}
\left.H_{n} a x+b\right] & =\sum_{i=0}^{n} a_{i}(a x+b)_{n-i} & \text { where } & (a x+b)_{n}=\prod_{r=0}^{n-1}\left(a x+b q^{r}\right) ; \\
H_{n}{ }^{*}[a x+b] & =\sum_{i=0}^{n} a_{i}(a x+b)_{n-i}^{*} \quad \text { where } & & (a x+b)_{n}{ }^{*} q^{n(n-1) / 2}=(b+a x)_{n} ; \\
H_{n}[x] & =\sum_{i=0}^{n} a_{i} q^{(n-i)(n-i-1) / 2} x^{n-i} ; & & H_{n}{ }^{*}[x]=\sum_{i=0}^{n} a_{i} q^{-(n-i)(n-i-1) / 2} x^{n-i} .
\end{aligned}
$$

Received August 18, 1967. Revised November 17, 1967.

* R. P. Boas and R. C. Buck, (Polynomial Expansions of Analytic Functions, Ergebnisse der Math., Vol. 19, 1958, pp. 44-45) call them Brenke polynomials and Waleed A. Al-Salam (a paper in press) calls them $q$-Appell polynomials and discusses their algebraic structure also. 
We shall use the following notations:

$$
\begin{aligned}
& {[x]=\left(q^{x}-1\right) /(q-1), \quad[x]_{s}=[x][x-:] \cdots[x-s+1] ;} \\
& {[x] !=[x][x-1] \cdots[1], \quad[0] !=1 ; \quad\left[\begin{array}{l}
x \\
s
\end{array}\right]=[x]_{s} /[s] !, \quad\left[\begin{array}{l}
x \\
0
\end{array}\right]=1 .}
\end{aligned}
$$

In analogy with Nielsen [2] we shall call the set of polynomials $\left\{H_{n}(x, q)\right\} \equiv$ $\left\{H_{n}(x)\right\}$ which satisfy $(5)$ to be " $(q, k)$-harmonic". If $\left[H_{n}(x), h_{n}\right]$ and $\left[K_{n}(x), k_{n}\right]$ are two $(q, k)$-harmonic sequences, then it is easy to prove that

(a) there exists a sequence of constants $\left\{h_{n}\right\}$ such that

(b)

$$
\begin{gathered}
H_{n}(x)=\sum_{i=0}^{n} h_{i} q^{k(n-i)(n-i-1) / 2} \frac{x^{n-i}}{[n-i] !}, \quad H_{n}(0)=h_{n} ; \\
D_{q}\left\{H_{n}[x]\right\}=H_{n-1}\left[q^{k+1} x\right], \quad(n=0,1,2, \cdots) ; \\
D_{q}\left\{H_{n}{ }^{*}[x]\right\}=H_{n-1}^{*}\left[q^{k-1} x\right],
\end{gathered}
$$

(c) $\sum_{n=0}^{\infty} H_{n}(x) t^{n}=e_{q, k}(x t) h(t)$ where

(d)

$$
\begin{gathered}
e_{q, k}(x)=\sum_{n=0}^{\infty} \frac{x^{n}}{[n] !} q^{k n(n-1) / 2} \text { and } h(t)=\sum_{n=0}^{\infty} h_{n} t^{n} ; \\
H_{n}[x+b]=\sum_{i=0}^{n} \frac{x^{i}}{[i] !} q^{k i(i-1) / 2} H_{n-i}\left[q^{k i} b\right] \\
H_{n}{ }^{*}[x+b]=\sum_{i=0}^{n} \frac{x^{i}}{[i] !} q^{-k i(i-1) / 2} H_{n-i}^{*}\left[q^{k i} b\right] \\
H_{n}[b+x]=\sum_{i=0}^{n} \frac{x^{i}}{[i] !} q^{(k+1) i(i-1) / 2} H_{n-i}\left(q^{k i} b\right), \\
H_{n}{ }^{*}[b+x]=\sum_{i=0}^{n} \frac{x^{i}}{[i] !} q^{(k-1) i(i-1) / 2} H_{n-i}\left(q^{(k-1) i} b\right) ;
\end{gathered}
$$

(e) there exists a unique sequence $\left\{\alpha_{n}\right\}$ such that for all $n$

$$
K_{n}(x)=\alpha_{0} H_{n}(x)+\alpha_{1} H_{n-1}(x)+\cdots+\alpha_{n} H_{0}(x) ;
$$

(f) $D_{q} H_{n}{ }^{*}(x)=H_{n-1}\left(q^{1-k} x\right)$ where $H_{n}{ }^{*}(x)=H_{n}{ }^{*}(x, q)=H_{n}(x, 1 / q)$;

(g) the expression

$$
A_{n}{ }^{k}=\sum_{s=0}^{n}(-1)^{s} H_{n-s}\left(q^{k s} x\right) K_{s}\left[q^{(n-s) k} x\right] q^{-k s(n-s)}
$$

is a constant, while the polynomials

$$
G_{n}{ }^{k}(x)=\prod_{r=1}^{n}\left(1+q^{r-k}\right)^{-1} \sum_{s=0}^{n} H_{n-s}\left(q^{(k-1) s} x\right) K_{s}\left[q^{(n-s) k} x\right]
$$

form a new $(q, k)$-harmonic sequence;

(h) if

$$
H_{n}[x]-H_{n}[-1+x]=K_{n-1}(x),
$$


then if only one of these $(q, k)$-harmonic sets is given the second is completely determined;

(i) if

$$
H_{n}[x]+H_{n}[-1+x]=K_{n}(x),
$$

then also if one of these is given the other can be completely determined.

As particular cases of these results we can get all the results obtained by Sharma and Chak [3] by just taking $k=0$ and those of Nielsen [2] by further taking the limiting case of the difference operator $D_{q}$ with $q \rightarrow 0$ i.e. the differential operator $D \equiv d / d x$.

2. $(q, k)-(\mathrm{I})$ and $(q, k)-(\mathrm{II})$-Regular Sequences (when " $a$ " is Not a Root of Unity). If the set of polynomials $\left\{H_{n}(x)\right\}$ satisfies the two functional relations $(n=0,1,2, \cdots)$

$$
D_{q}\left\{H_{n}(x)\right\}=H_{n-1}\left(x q^{k}\right) \text { and } H_{n}\left[a x+b / q^{k n}\right]=\tau_{n} H_{n}(x),
$$

then we shall call them $(q, k)$-(I)-regular sequences. If " $a$ " is not 0 or 1 it is easy to prove that:

The necessary and sufficient condition for a $(q, k)$-harmonic sequence $\left\{H_{n}(x)\right\}$ to be $(q, k)$-(I)-regular is

$$
H_{n}\left[b / q^{k n}\right]=a^{n} H_{n}(0) ; \quad n=0,1,2, \cdots .
$$

If we expand the left-hand side of (2.2) and equate, we easily get

$$
h_{n}=\frac{h_{0} b^{n} \Delta_{n}{ }^{k}(a, q)}{(a-1)\left(a^{2}-1\right) \cdots\left(a^{n}-1\right)}
$$

where

$$
\Delta_{n}{ }^{k}(a, q)=\left|\begin{array}{llllll}
\frac{1}{q^{k}[1] !} & (1-a) & 0 & 0 & \cdots & 0 \\
\frac{q^{k+1}}{\left(q^{2 k}\right)^{2}[2] !} & \frac{1}{q^{2 k}[1] !} & \left(1-a^{2}\right) & 0 & \cdots & 0 \\
\frac{q^{3(k+1)}}{\left(q^{3 k}\right)^{3}[3] !} & \frac{q^{k+1}}{\left(q^{3 k}\right)^{2}[2] !} & \frac{1}{q^{3 k}[1] !} & \left(1-a^{3}\right) & \cdots & 0 \\
\cdot & \cdot & \cdot & \cdot & \cdots & \cdot \\
\frac{q^{(k+1) n(n-1) / 2}}{\left(q^{n k}\right)^{n}[n] !} & \frac{q^{(k+1)(n-1)(n-2) / 2}}{\left(q^{n k}\right)^{n-1}[n-1] !} & \cdot & \cdot & \cdots & \frac{1}{q^{n k}[1] !}
\end{array}\right| .
$$

We can also define another set of polynomials $\left\{H_{n}(x)\right\}$ satisfying, for $n=0,1,2, \cdots$,

$$
D_{q}\left\{H_{n}(x)\right\}=H_{n-1}\left(x q^{k}\right) \text { and } H_{n}\left[b / q^{k n}+a x\right]=\tau_{n} H_{n}[x]
$$

and call them $(q, k)$-(II)-regular sequences. If " $a$ " is not a root of unity we can easily get the necessary and sufficient condition [3] for this set also.

3. $(q, k)-(\mathrm{T})$-Regular Sequences and the Case when " $a$ " is a Root of Unity. For both $(q, k)$-(I)-regular and $(q, k)$-(II)-regular sequences we see that $h_{1}, h_{2}, \cdots$ 
are finite only if " $a$ " is not a root of unity. If, however, $a^{r}=1[r \equiv 0(\bmod p)]$, then it is easy to see that an infinite sequence of polynomials $\left\{H_{n}(x)\right\}$ does not exist which satisfies (2.1) or (2.4). In order to be able to discuss the case when " $a$ " is a root of unity we take the two functional equations as given in (3.1) below (instead of (2.1) and (2.4)) introducing special triangular matrices $T$ and $T^{\prime}$ of nonzero numbers similar to the ones introduced in [3] and get all the results obtained in [3], for $(q, k)$-(T)-regular sequences defined by $(n=0,1,2, \cdots)$

$$
D_{q}\left\{H_{n}(x)\right\}=H_{n-1}\left(q^{k} x\right) \text { and } H_{n}{ }^{T}\left(a x+b / q^{k n}\right)=\tau_{n} H_{n}(x) .
$$

Following [3] we can easily get the results for these more general cases. If we take $k=0$ we get the results given in [3].

West Virginia University

Morgantown, West Virginia 26506

1. L. Carlitz, "Some polynomials related to Theta functions," Ann. Mat. Pura Appl., (4), v. 41, 1956, pp. 359-373. MR 17, 1205; ibid., Duke Math.J., v. 24, 1957, pp. 521-527. MR 19, 849.

2. N. NIELSEN, Traité Elémentaire des Nombres de Bernoulli, Gauthier-Villars, Paris, 1923.

3. A. Sharma \& A. M. ChaK, "A basic analogue of a class of polynomials," Riv. Mat. Univ. Parma, v. 5, 1954, pp. 325-337. MR 17, 150 .

4. M. WARD, "A certain class of polynomials," Ann. of Math., v. 31, 1930, pp. 43-51. 